as well as histologically assessed disease severity (using the Gomes scoring system).

Results Arachidonic acid (AA), but not eicosapentaenoic acid (EPA), derived eicosanoids (prostaglandin (PG) $\mathrm{E}_{2}, \mathrm{PGD}_{2}$, thromboxane $\mathrm{B}_{2}$, 5-hydroxy-eicosatetraenoic acid (HETE), 11-HETE, 12-HETE and 15 -HETE), were significantly $(p<0.001)$ higher in inflamed than non-inflamed mucosa and their concentrations correlated to histological severity.

Conclusion There is an upregulation of AA derived inflammatory mediators in UC. This research suggests new eicosanoid targets for research and therapeutic intervention.

Disclosure of Interest None Declared

\section{PTU-067 DUAL-ENERGY X-RAY ABSORPTIOMETRY UTILITY IN INFLAMMATORY BOWEL DISEASE: BSG, FRAX, NOGG OR NICE?}

doi:10.1136/gutjnl-2013-304907.159

'S Ah-Moye, 1."D Chan, ${ }^{1} \mathrm{~J}-Y$ Kang, ${ }^{1} \mathrm{R}$ Pollok, ${ }^{1} \mathrm{P}$ Neild, ${ }^{2} \mathrm{~K}$ Moss, ${ }^{1} \mathrm{~A}$ Poullis. ${ }^{1}$ Department of Gastroenterology; ${ }^{2}$ Department of Rheumatology, St George's Hospital, London, UK

Introduction Patients with inflammatory bowel disease (IBD) have a $40 \%$ higher risk of osteoporotic fractures than the general population. In 2007 the British Society of Gastroenterology (BSG) produced Guidelines for Osteoporosis in Inflammatory Bowel Disease and Coeliac Disease. In 2008 the World Health Organization created the Fracture Risk Assessment (FRAX) tool, to assess the risk of fracture in an individual aged 40 years or over. The National Osteoporosis Guideline Group (NOGG) was subsequently established to provide guidelines for the management of patient's after assessment with FRAX. In August 2012 the National Institute for Health and Clinical Excellence (NICE) published their recommendations. This study aims to compare these guidelines in IBD patients and recommendations for dual-energy X-ray absorptiometry (DXA) scan.

Methods Over a four-month period, IBD patients attending the Gastroenterology Departments' of St. George's and Queen Mary's Hospital were identified. Convenience sampling was used; all IBD patients encountered in these clinics were asked to participate. Patients were asked to complete a questionnaire, gathering information required for the BSG, NOGG and NICE osteoporosis guidelines. The BSG and NICE guidance, were used in all patients. Additional assessment with NOGG guidance were used in patients 40 years and over. The recommendation for DXA scan or not were noted.

Results 153 patients were included in the study. 73 were men and 80 were women. The mean age was 42 years with an age range of 17 to 82 years.

The BSG guidelines were applicable to the entire patient group. 100 patients $(65.3 \%)$ were recommended a DXA scan and 53 (34.6\%) were not. The NICE guidelines were applicable to the whole patient group; with 37 (24.1\%) recommended a DXA scan and 83 (54.2\%) not.

In patients over 40 years of age there was good concurrence between all guidelines recommending a DXA scan. The BSG guidance recommended 44 patients $(63.7 \%)$, NOGG recommended 42 patients $(60.8 \%)$ and NICE recommended 42 patients $(60.8 \%)$. In the group less than 40 years of age, the BSG guidance recommended 56 patients $(66.6 \%)$ and NICE recommended $28(33.3 \%)$ to have a DXA scan.

Conclusion There are a number of assessment tools available to assess the risk of osteoporosis in IBD patients and identify those who should have a DXA carried out. Our study has shown that in patients 40 years and over there is a reasonable concurrence between all of these assessments. For the age group less than 40 years there appeared to be less concordance. These assessment tools need to be compared further to DXA scan results, to establish the best assessment tool for IBD patients and when to commence osteoporosis treatment.

Disclosure of Interest None Declared

\section{PTU-068 PATIENT AWARENESS OF IMMUUNISATION GUIDELINES IN INFLAMMATORY BOWEL DISEASE}

doi:10.1136/gutjnl-2013-304907.160

${ }^{1} \mathrm{~K}$ Amir, ${ }^{1,} \mathrm{D}$ Chan, ${ }^{1} \mathrm{~J}-\mathrm{Y}$ Kang, ${ }^{1} \mathrm{R}$ Pollok, 'P Neild, 'A Poullis. 'Department of Gastroenterology, St George's Hospital, London, UK

Introduction Immunomodulator agents are commonly employed in the management of inflammatory bowel disease (IBD). These can increase the risk for opportunistic infections. This study aims to assess patient awareness for the need for appropriate immunisations, as outlined in published guidelines by the European Crohn's and Colitis Organisation (ECCO) [1].

Methods Over a four-month period, IBD patients attending the Gastroenterology Departments' of St. George's Hospital and Queen Mary's Hospital were identified. Convenience sampling was used; all IBD patients encountered in these clinics were asked to participate. Patients were asked to complete a questionnaire, gathering information about their disease and immunisation awareness.

Results 135 patients participated in the study. 73 patients were male. Mean age was 43 years (with the range being $19-82$ years). 53 patients had ulcerative colitis, 73 had crohn's disease and 9 were unsure of their diagnosis. Mean time since diagnosis was 8 years.

18 patients $(13.3 \%)$ were currently receiving no drug therapy. 34 (25.1\%) were being treated with immunomodulator agents (azathioprine, 6-mercaptopurine, methotrexate or cyclosporin). 4 patients $(2.9 \%)$ were solely receiving biologics (infliximab or adalimumab), and 46 patients (34.0\%) were on 5-aminosalicylic acids (5-ASAs) only. 27 patients (20\%) were on a combination of thiopurines and 5-ASA therapy, and 6 patients (4.4\%) were on a combination of infliximab, azathioprine and 5-ASA. Prednisolone therapy had been taken at some stage of treatment by 72 patients $(53.3 \%)$.

The majority of patients were not aware if they had been screened at diagnosis for the specific infections outlined by ECCO. Of the total 135 patients the following were aware that they had undergone screening: 4 (2.9\%) for varicella zoster virus (VSV), 1 $(1.9 \%)$ for hepatitis B virus (HBV), 3 (2.2\%) for human immunodeficiency virus (HIV), 2 (1.4\%) for hepatitis $\mathrm{C}$ virus (HCV) and 15 (11.1\%) for tuberculosis (TB).

Of the 135 patients: 4 (2.9\%) recalled been offered immunisation against VZV, 16 (11.8\%) against HBV, 51 (37.7\%) against influenza, $33(24.4 \%)$ against pneumococcus and none against human papilloma virus.

$98(72 \%)$ patients had not had any discussions with their General Practitioner about immunisations, 45 (33\%) had read the immunisation advice distributed to all IBD patients from clinic.

Conclusion Our findings show that IBD patients are largely unaware of recommendations by ECCO regarding immunisation. Patient awareness needs to be increased regarding opportunistic infections and prevention with immunisations. A patient awareness campaign to educate IBD patients has been started in our clinics.

Disclosure of Interest None Declared

\section{REFERENCE}

1. European Crohn's and Colitis Organisation (ECCO) guidelines (2009). http://www. ecco-ibd.tv/index.php/publications/ecco-guidelines

\section{PTU-069 THE EFFECT OF FAECAL CALPROTECTIN ON THE USE OF COLONOSCOPY AT LANCASHIRE TEACHING HOSPITALS NHS FOUNDATION TRUST}

doi:10.1136/gutjnl-2013-304907.161

1."E Shuttleworth, 'A Poppleton, 'E Lim, 'A Sharma. 'Dept of Gastroenterology, Lancashire Teaching Hospitals NHS Foundation Trust, Preston, UK

Introduction Faecal calprotectin is a sensitive measure of neutrophilic intestinal inflammation; use in gastroenterological screening 
has been proposed, reducing invasive and costly lower gastrointestinal (GI) endoscopy, particularly when functional disorders are suspected [1]. We reviewed all faecal calprotectin results and available patient records over a six month period to determine whether a normal faecal calprotectin resulted in subsequent avoidance of lower GI endoscopy.

Methods The paper and/or electronic medical records for patients with calprotectin requests over a 6 month period were accessed and reviewed by a Specialty Trainee in gastroenterology at Lancashire Teaching Hospitals NHS Foundation Trust. Demographic data, symptoms, and recent or subsequent lower GI endoscopy were recorded. Endoscopy was considered 'spared' if the diagnosis was anticipated to be functional and a normal faecal calprotectin resulted in avoidance of lower GI endoscopy; or in patients with known inflammatory bowel disease (IBD) where the notes intimated a normal calprotectin would result in avoidance of further endoscopy.

Results 122 patients (73 female, mean age 41yrs, range 16-89yrs) had faecal calprotectin requests sent from the Royal Preston Hospital and Chorley and South Ribble Hospital. 4 samples were not received or processed. $90.2 \%$ were outpatient requests, and the most common indications were loose stools $(54.1 \%)$ and abdominal pain (28.7\%). 45 samples (36.9\%) had a raised calprotectin $(\geq 51 \mu \mathrm{g} / \mathrm{g})$, of which 20 patients had known IBD. In those with a normal calprotectin $(n=73), 56$ had an unclear diagnosis of which $4(7.1 \%)$ eventually went on to have lower GI endoscopy with no major findings, and 20 patients $(35.7 \%$ ) had previously undergone lower GI endoscopy. In 13 patients there were insufficient records available to be able to comment. According to the criteria mentioned 35 patients were spared lower GI endoscopy within the 6 month period.

Conclusion Over a 6 month period 35 lower GI endoscopies were avoided representing both a reduction in patient exposure to the risks of endoscopy and a significant financial saving. Our results suggest that faecal calprotectin is a valuable and cost effective resource in helping to exclude significant organic pathology in selected patients given its proven high negative predictive value in excluding gastrointestinal inflammation [2].

Disclosure of Interest None Declared

\section{REFERENCES}

1. Licata A et al. J Clin Gastroenterol. 2012; 46:504-8.

2. Meucci G et al. Dig Liver Dis. 2010; 42:191-5

\section{PTU-070 SELECTING THE MOST COST-EFFECTIVE MODEL OF CARE FOR DELIVERING BIOLOGICAL AGENTS AS MAINTENANCE THERAPY IN PATIENTS WITH CROHN'S DISEASE}

\author{
doi:10.1136/gutjnl-2013-304907.162
}

1."E Lougher, ${ }^{2} \mathrm{M}$ C Allison, ${ }^{3} \mathrm{~K}$ Hodson, ${ }^{1} \mathrm{M}$ Pugh. ${ }^{1}$ Pharmacy; ${ }^{2}$ Gastroenterology, Aneurin Bevan Local Health Board, Newport; ${ }^{3}$ Cardiff School of Pharmacy and Pharmaceutical Science, Cardiff University, Cardiff, UK

Introduction The introduction of anti-TNF alpha monoclonal antibodies in 1999 has revolutionised the management of inflammatory bowel disease (IBD). A significant increase in gross spend on biological agents in the management of Crohn's disease has occurred since the implementation of NICE guidance in 2002. The unplanned nature of the service expansion and evolution has led to a wide variation in service delivery. Within the Aneurin Bevan Local Health Board ( $\mathrm{ABHB}$ ) the approximate doubling in the gastroenterology spend on biologics (between 2010 and 2011) prompted a review of current services and an investigation of other potential models of care for delivering the service.

Methods A service evaluation for both adalimumab and infliximab (IFX) including: an assessment of the current services from a patient's perspective (study 1), identifying and exploring models of care for delivering the service (study 2) and evaluating the costs associated with each model of care (study 3 ) was undertaken. Study 1 comprised face-to-face semi-structured, tape recorded patientinterviews, which were transcribed verbatim and then thematically analysed. Study 2 utilised a number of methods to identify keyinformants at various secondary care sites to participate in telephone semi-structured interviews, models identified were compared and contrasted. Study 3 identified and compared the costs of current models within $\mathrm{ABHB}$ with viable models identified in study 2.

Results The results revealed overall satisfaction with the IBD services within $A B H B$ and with the service provided by Healthcare at Home Ltd. Patients were complementary of the IBD team and the telephone help line. Nonetheless areas for improvement with regards to the infusion facilities were identified by the IFX group. Study 3 identified four models of care: IFX prepared in pharmacy, IFX prepared by a specialist nurse, IFX at home and adalimumab at home. For standard dosing ( $79 \mathrm{~kg}$ patient-average IFX patient weight at $\mathrm{ABHB}$ ) annual costs were $£ 12,237, £ 12,314, £ 10,254$ and $£ 9,156$ respectively, inclusive of pharmacy production time, nursing time and active drug and exclusive of hospital facilities. Vial sharing would reduce the cost of models one and two, however would require complex re-organisation to facilitate "pairing" patients Study 3 identified adalimumab via Healthcare at Home as being the most cost-effective model.

Conclusion Where clinically appropriate adalimumab via Healthcare at Home Ltd is recommended for this group of patients within $\mathrm{ABHB}$, with IFX via a home care company as second line. Work should be done to improve the current infusion facilities. Future work should include reviewing the potential of setting up a biologics unit shared between specialities.

Disclosure of Interest None Declared

\section{PTU-071 CAUSE OF DEATH IN THE EXETER INFLAMMATORY BOWEL DISEASE (IBD) POPULATION}

doi:10.1136/gutjinl-2013-304907.163

1,"E Wesley, 'B Beddoes, 'A Forbes, 'M Clarke, 'T K Daneshmend, 'T Ahmad. 'Gastroenterology, Royal Devon and Exeter Hospital, Exeter, UK

Introduction Recent meta-analyses of population based IBD mortality studies have shown no increase in standardised mortality ratio for ulcerative colitis (UC) ${ }^{1}$, but a small increase for Crohn's disease $(C D)^{2} .26-50 \%$ of deaths in CD patients were related to the underlying IBD diagnosis ${ }^{2}$. The aims of this study were to investigate the death cause distribution in a well defined IBD population and to establish the proportion of deaths related to the underlying diagnosis or treatment.

Methods Retrospective case note review to establish cause of death of all patients on the Exeter IBD database who died in the 9yr period until 31.12.2011. Cases were reviewed by 2 clinicians to establish cause of death and ascertain whether it was related to the underlying diagnosis or treatment.

Results 143 deaths, 82 male. 87 had UC, 46 CD and 10 IBD unclassified. Mean age at diagnosis was $58(11-94)$. Mean duration of disease at time of death was 17 years (0-61). The median age of death was 72.5 and 79 years for CD and UC respectively $(P=0.001$, $95 \%$ CI $-13--3$ years).

The underlying disease or treatment was implicated in the cause of death in $35 / 143$ (23\%) patients and was more common in CD compared to UC patients ( 37 vs. $18 \%, P=0.03$ ). There was no change over time in the proportion of related deaths.

$13 / 143(9 \%)$ had a diagnosis of colorectal cancer at the time of death; in 8 this was the primary cause of death. 8 had Primary Sclerosing Cholangitis, all of whom died of related complications. 8 patients had a haematological malignancy including 3 patients with colonic lymphomas, 1 with chronic myeloid leukaemia, 2 with nonHodgkin's lymphoma and 1 with peripheral T cell lymphoma. Half of these patients had previously been prescribed thiopurines. 